\title{
Aproximación al tratamiento de aguas residuales del lavado del café con las microalgas Parachlorella kessreli y Desmodesmus armatus
}

\author{
An Approach for Treating Wet Coffee Processing Wastewater Using \\ Microalgae Parachlorella kessreli and Desmodesmus armatus
}

\author{
Iván Alberto Sandoval Salazarac, Mohamed Toufic Darwich Cedeño ${ }^{\mathrm{b}}$, María Del \\ Rosario Castañeda ${ }^{\text {ad }}$, Wilmer Andrés Torres Munara, Luis Carlos Montenegro \\ Ruiz ${ }^{\text {be }}$
}

\footnotetext{
a Grupo de Investigación Yamboró, Servicio Nacional de Aprendizaje (SENA), Pitalito, Huila, Colombia

${ }^{\text {b }}$ Laboratorio de Cultivo de Algas, Facultad de Ciencias, Universidad Nacional de Colombia, Colombia.

chttps://orcid.org/0000-0002-5679-7798

d https://orcid.org/0000-0002-6171-7756

eIcmontenegror@unal.edu.co| https://orcid.org/0000-0002-6662-1833
}

\section{RESUMEN}

Colombia es uno de los mayores productores de café en el mundo, con un mercado que superó los 13,9 millones de sacos en 2020. Sin embargo, en el proceso de beneficio húmedo del café se emplean grandes cantidades de agua, aproximadamente $4.154 .354 \mathrm{~m}^{3}$, en las fases de despulpado, lavado y transporte del grano, generando aguas residuales contaminadas que tienen poco tratamiento antes de ser vertidas a las fuentes hídricas. En el presente trabajo se buscó aislar especies de microalgas que crezcan en estos ambientes, utilizar estas especies en procesos de depuración de aguas residuales del proceso de lavado del café y buscar una aplicación

Citation: Sandoval-Salazar, I. A., Darwich-Cedeño, M. T., Castañeda, M. R., Torres-Munar, W. A. y Montenegro-Ruiz, L. C. (2021). Aproximación al tratamiento de aguas residuales del lavado del café con las microalgas Parachlorella kessreli y Desmodesmus armatus. Mutis, 11(2), 32-43. https:// doi.org/10.21789/22561498.1755

Recibido: 19 de mayo de 2021. Aceptado: 30 de junio de 2021.

Copyright: $\odot 2021$. Sandoval-Salazar, I. A., Darwich-Cedeño, M. T., Castañeda, M. R., Torres-Munar, W. A. y Montenegro-Ruiz, L. C. (2021). This is an openaccess article, which permits unrestricted use, distributions and reproduction in any medium, provided the original author and source are credited.

Competing Interests: The authors have no conflict of interest. de la biomasa resultante. Para la obtención de las cepas de microalgas se utilizaron técnicas de agotamiento. Con el fin de evaluar su capacidad de depuración, estas fueron cultivadas en agua residual de diferentes concentraciones bajo condiciones de laboratorio, determinando la remoción de materia orgánica. Adicionalmente, se caracterizó bioquímicamente la biomasa algal para determinar posibles usos biotecnológicos o industriales. Se encontró dos Chloroficeaes cocales, Parachlorella kessreli y Desmodesmus armatus, las cuales demostraron una alta eficiencia en la remoción de nitrógeno total (> 50 \%) y fósforo total 
(> 70 \%), así como una disminución significativa de la DQO (> 70 \%) en los mejores tratamientos. Finalmente, se encontró para Parachlorella kessreli alta producción de lípidos y para Desmodesmus armatus alta producción de proteína.

Palabras clave: café, aguas residuales, Parachlorella, Desmodesmus, depuración de agua, microalgas.

\section{ABSTRACT}

Colombia is one of the largest coffee producers in the world, with a market that exceeded 13.9 million bags in 2020. However, wet coffee processing requires large amounts of water -approximately 4,154, $354 \mathrm{~m}^{3}-$ in the pulping, washing and transportation phases, generating contaminated wastewater that has little treatment before being discharged to water sources. Hence, this work sought to isolate microalgae species that grow in these environments to use them wastewater treatment processes from the coffee washing process and seek an application of the resulting biomass. Depletion techniques were used to obtain the microalgae strains. In order to evaluate their purification capacity, the microalgae were cultivated in wastewater at different concentrations under laboratory conditions, determining the removal of organic matter. Additionally, the algal biomass was biochemically characterized to determine possible biotechnological or industrial uses. Two cocal Chloroficeaes, Parachlorella kessreli and Desmodesmus armatus, were found after this process. Both of them showed high efficiency in removing total nitrogen $(>50 \%)$ and total phosphorus $(>70 \%)$ while decreasing COD by more than $70 \%$ in the best treatments. High lipid production was found for $P$. kessreli, and high protein production for $D$. armatus.

Keywords: Coffee, wastewater, Parachlorella, Desmodesmus, water purification, microalgae.

\section{INTRODUCCIÓN}

El departamento del Huila es uno de los mayores productores de café en Colombia, con 2,5 millones de sacos producidos por año y 147.275 hectáreas sembradas en 35 de sus 37 municipios. El café producido en este departamento es reconocido a nivel mundial por la diversidad de sabores en taza, aroma y calidad, además de ser el principal factor económico y social de la región. A pesar de su importancia, el proceso de beneficio húmedo del café emplea grandes cantidades de agua en las etapas de despulpado, lavado y transporte del grano, registrando consumos aproximados de $4.154 .354 \mathrm{~m}^{3}$ que equivalen a $37,2 \mathrm{~L} / \mathrm{kg}$ de café pergamino seco (Rodríguez et al., 2015). El proceso, a su vez, genera grandes volúmenes de aguas residuales que contaminan las aguas y los suelos de las zonas circundantes a las áreas de producción. Debido a los altos volúmenes de agua residual se hace imposible aplicar tratamientos antes de su vertimiento a cielo abierto, lo que deteriora las fuentes hídricas en las épocas de cosecha. En cuanto al grado de contaminación causado por las aguas mieles del café en un cuerpo de agua, se ha determinado que la demanda biológica de oxígeno (DBO5) aproximada es de 25.109 t, los sólidos totales disueltos alcanzan $23.830 \mathrm{t}$ y el $\mathrm{DQO}$ registra un valor de $55.000 \mathrm{mg} / \mathrm{L}$ (Rodríguez et al., 2015).

En ese contexto, los impactos ambientales generados por el procesamiento del café se relacionan con la pérdida de la biodiversidad en aguas y suelos, una disminución en la calidad de vida de los productores, quienes reportan problemas de salud por el consumo de aguas contaminadas, la presencia de malos olores, la aparición de insectos vectores, y un menor rendimiento de los suelos, ocasionado por el alto contenido de materia orgánica y acidez que altera el microbiota. Por lo anterior, la mitigación de los impactos ambientales causados por las aguas residuales del café mediante el uso de microorganismos nativos como microalgas y cianobacterias se convierte en una alternativa viable y con potencial para el tratamiento terciario de este subproducto del café en el departamento del Huila.

Durante los últimos años, las microalgas y cianobacterias se han utilizado como sistemas biológicos alternativos al tratamiento de aguas residuales, en gran parte por su capacidad de combinar su metabolismo autótrofo con el heterótrofo, con lo cual consumen sales e iones en presencia de luz, a la 
vez que pueden consumir moléculas orgánicas. Investigaciones desarrolladas por Li Wu et al. (2018), quienes cultivaron la cianobacteria Scytonema javanicum en aguas residuales sintéticas artificiales, determinaron el potencial remediador de nitrógeno y fosfatos de este organismo. Por su parte, Lynch et al. (2015) aislaron siete especies nativas de cianobacterias y un alga verde con el fin de evaluar su capacidad de eliminación de nutrientes, logrando con ello eliminar totalmente el fosfato y el amonio, y obteniendo al final un alto contenido de biomasa. Gorain et al. (2019) evaluaron la eliminación de nitrógeno y fosfato en aguas residuales agrícolas utilizando la cianobacteria Anabaena sphaerica y A. variabilis durante 30 días en un sistema de cultivo semicontinuo, encontrando que cerca del $90 \%$ de $\mathrm{N}$ y $\mathrm{P}$ fueron eliminados, además de que la biomasa producida reportaba altos contenidos de ácidos grasos esenciales con potencial para su uso en la producción de nutracéuticos.

Sumado a lo anterior, se ha evaluado también el uso conjunto de microalgas y cianobacterias (Oscillatoria $s p$.) para la eliminación de nitrógeno y fósforo inorgánico en aguas residuales, encontrando que es posible eliminar 36 mg de $\mathrm{N}$ y 0,35 mg de P por día y generar una producción de biomasa de $0,15 \mathrm{mg} /$ día (Arias et al., 2017). Otras algas y cianobacterias que han dado buenos resultados en depuración son Phomidium $s p$. capaz de eliminar $100 \%$ de ortofosfato, $87 \%$ de nitrato, $68 \%$ de fosfato total y $48 \%$ de ion amonio (Cañizares-Villanueva et al., 1994), Chlorella vulgaris y Scenedesmus acutus, empleadas para la remoción de cromo en aguas de curtiembres (Ardila et al., 2017).

Para aguas residuales del proceso productivo de café no se han realizado tratamientos con algas, hasta donde conocemos, aunque sí se ha avanzado en la investigación con otros microorganismos que resultan ser eficientes en la depuración de las aguas finales del proceso. Entre los trabajos que se destacan por dar resultados positivos está el de Ashenafi et al. (2021), quienes evaluaron a escala laboratorio la eficiencia de las bacterias Pseudomona fluorescence y Escherichia coli, obteniendo en 144 horas reducción de DBO5, DQO y TS. De otro lado, Pires et al. (2021) desarrollaron un protocolo para la obtención de microorganismos con potencial para la remoción de la carga orgánica a partir de la microbiota autóctona de las aguas residuales de la producción de café, logrando efectividad en un consorcio bacteriano compuesto por Serratia marcescens, Corynebacterium flavescens y Acetobacter indonesiensisk, con el que se logró disminuir 85 \% la DBO5, 60 \% la DQO y 80 $\%$ el fósforo y el nitrógeno. Resultados similares se alcanzaron con cepas bacterianas autóctonas aisladas de las aguas residuales del despulpado de la cereza de café (Enterobacter ludwigii, Bacillus cereus, Enterobacter aerogenes y Enterobacter cloacae), disminuyendo hasta en $40 \%$ la DQO (Jenifer et al., 2020). También se han realizado trabajos con los hongos Aspergillus niger y Aspergillus flavus, microorganismos aislados de las mismas aguas residuales, alcanzando una reducción de hasta $80 \%$ de DQO y DBO (Navitha \& Kousar, 2018).

Teniendo en cuenta lo anterior, el presente trabajo llevó a cabo una exploración de especies de microalgas aisladas de los procesos de producción de café con alta capacidad para depurar las aguas residuales $y$, a la vez, hacer posible que la biomasa algal producida tenga un valor agregado para su utilización en procesos tanto biotecnológicos como industriales.

\section{MATERIALES Y MÉTODOS}

\section{Colecta de algas y obtención de cepas}

Las muestras analizadas provienen de fincas cafeteras del municipio de Isnos (Huila, Colombia). Se realizó toma de muestras en los tanques de almacenamiento de pulpa de café, mucilago y en los procesos de filtrado de aguas residuales del proceso de producción de café. Las muestras de agua se recolectaron en tubos Falcon de $50 \mathrm{~mL}$ para luego ser etiquetadas y conservadas en oscuridad a temperatura ambiente hasta el momento de su procesamiento.

Para la obtención de cepas unialgales se utilizó la técnica de rayado en agar por agotamiento. Como medio de cultivo se utilizó medio básico Bold (BBM) enriquecido con agar-agar 1,5\%. En esta fase se hicieron tantos repiques como fueron necesarios hasta alcanzar una cepa unialgal (Andersen, 2005).

\section{Identificación molecular de las cepas}

Para la identificación de las cepas se aisló ADN siguiendo el protocolo del kit ZYMO Research QuickDNA TM Plant/Seed Micropep (ZYMO Research Corp., 
Irvine, Estados Unidos, catálogo D6020). Para comprobar la obtención de ADN se realizó electroforesis en gel de agarosa a 1,5\%, utilizando como marcador buffer de carga e HydraGreen ${ }^{\text {TM }}$ Safe DNA Dye-Thomas Scientific. Para las cepas se amplificó el gen 18 s con los cebadores 18s F (AACCTGGTTGATCCTGCCAG) y 18s R (CACCAGACTTGCCCTCCA).

Todas las reacciones se realizaron en un termociclador MyCycler ${ }^{\mathrm{TM}}$ (Bio-Rad laboratorios Inc., Hercules, CA, EE. UU.) en alícuotas de $20 \mu \mathrm{L}$ que contenían 10 $\mu \mathrm{L}$ de PCR mix - 100 2X (Corpogen, Colombia), $1 \mu \mathrm{L}$ de cebador $F, 1 \mu \mathrm{L}$ de cebador $\mathrm{R}$ (cada cebador a una concentración de $10 \mu \mathrm{M}), 0,5 \mu \mathrm{L}$ de ADN y agua grado biología molecular, hasta alcanzar un volumen final de $20 \mu \mathrm{L}$. El programa de PCR fue 94 ㅇ C durante 5 minutos, seguidos de 35 ciclos de 94 ㅇ durante $1 \mathrm{~min}, 55$ oC por 1 s y 72 ㅇ C durante $3 \mathrm{~min}$, así como una extensión final a $72 \stackrel{\circ}{\circ}$ durante $10 \mathrm{~min}$.

La limpieza de los productos de PCR se realizó con el Kit DNA Clean \& Concentration TM-5 (ZYMO Research Corp., Irvine, Estados Unidos, catálogo D4013). La electroforesis se corrió en gel de agarosa al $1 \%$ con marcador HydraGreen ${ }^{\mathrm{TM}}$ Safe DNA Dye-Thomas Scientific por aproximadamente una hora, con voltaje de 100 V. Finalmente, se observó el resultado en un documentador de geles (Gel Doc ${ }^{\mathrm{TM}}$ XR+, BioRad).

Los productos purificados de PCR fueron enviados a la casa comercial (SSigMOL, Instituto de Genética, Universidad Nacional, Colombia) para el respectivo secuenciamiento. Las secuencias se editaron usando el programa Sequencher 5.1 (Gene Code Corporation, 2017). Las comparaciones de secuencias se llevaron a cabo utilizando las bases de datos BLAST del National Center for Biotechnology Information.

\section{Caracterización bioquímica de las cepas seleccionadas}

Las dos cepas fueron cultivadas en medio BBM en un volumen de $1 \mathrm{~L}$, las condiciones de cultivo fueron irradiancia de $300 \mu \mathrm{mol}$ de fotones $\mathrm{m}^{-2} \mathrm{~s}^{-1}$, fotoperíodo de 12:12 horas luz/oscuridad, $25 \stackrel{\circ}{\circ}+/-3$ o $C$ y burbujeo permanente con aire del ambiente. La biomasa se recolectó en fase estacionaria, siendo deshidratada a baja temperatura.

La determinación de proteínas se realizó por el método de Bradford (1976), para lo cual se pesó aproximadamente 0,01 g de biomasa liofilizada en tubos Falcon de $15 \mathrm{ml}$, se adicionó $6 \mathrm{ml}$ de buffer de fosfato de sodio [0,02 M; pH 6] (Melgarejo, 2010) y perlas de sílice (para asegurar el rompimiento celular). Esta muestra se pasó por vórtex durante $5 \mathrm{~min}$ y se centrifugó a $6.000 \mathrm{rpm}$ por $5 \mathrm{~min}$. Se extrajo 0,8 $\mathrm{ml}$ de sobrenadante, el cual fue dispuesto en tubo Epppendorf de $2 \mathrm{ml}$, añadiendo $0,2 \mathrm{ml}$ de reactivo de Bradford (Bio-Rad 2006) y agitando fuertemente en un vórtex por un minuto, para luego incubar por cinco minutos y medir la absorbancia a $595 \mathrm{~nm}$. Cada mezcla de reacción se realizó por triplicado.

La determinación de carbohidratos se realizó por el método de DuBois (DuBois et al., 1956; Gerchakov \& Hatcher 1972; Melgarejo 2010), para lo cual se pesó aproximadamente $0,01 \mathrm{~g}$ de biomasa liofilizada en tubos Falcon de $15 \mathrm{ml}$, agregando $6 \mathrm{ml}$ de $\mathrm{H}_{2} \mathrm{O}$ destilada y desionizada y perlas de sílice (para asegurar el rompimiento celular). La muestra se pasó por vórtex durante 5 min y se centrifugó a $6.000 \mathrm{rpm}$ por $5 \mathrm{~min}$. Se extrajo $210 \mu \mathrm{l}$ de sobrenadante, el cual fue dispuesto en tubo Eppendorf de $2 \mathrm{ml}$, posteriormente añadiendo $0,2 \mathrm{ml}$ de fenol al $80 \%$ y $1 \mathrm{ml}$ ácido sulfúrico $\left(\mathrm{H}_{2} \mathrm{SO}_{4}\right)$, para luego agitar la muestra fuertemente en un vórtex por un minuto y dejar enfriar a temperatura ambiente y en oscuridad (el compuesto que se forma es estable por 36 horas). Se midió la absorbancia a $485 \mathrm{~nm}$. Cada mezcla de reacción se realizó por triplicado.

La determinación de lípidos se realizó por el método de Sulfofosfovainillina (Byreddy et al., 2016; Cheng et al., 2011; Mishra et al., 2014). Se pesó aproximadamente $0,01 \mathrm{~g}$ de biomasa liofilizada en tubos Falcon de $15 \mathrm{ml}$, se adicionó $2 \mathrm{ml}$ de cloroformo:metanol [1:1] y perlas de sílice (para asegurar el rompimiento celular), pasando la muestra por vórtex durante $5 \mathrm{~min}$ y centrifugando a $6.000 \mathrm{rpm}$ por $5 \mathrm{~min}$. Se extrajo $60 \mu \mathrm{l}$ de sobrenadante, siendo este dispuesto en tubo Eppendorf de $2 \mathrm{ml}$, para luego añadir 300 $\mu \mathrm{l}$ de ácido sulfúrico $\left(\mathrm{H}_{2} \mathrm{SO}_{4}\right)$ concentrado y someter la muestra a baño María de 90 ㅇ C durante $20 \mathrm{~min}$, para así evaporar los solventes y propiciar la reacción de hidrolisis. La muestra fue luego enfriada en un congelador durante 10 min y se agregó $500 \mu \mathrm{l}$ de ácido fosfórico al $17 \%$ - Vainillina [0,2 g de vainillina/ $\mathrm{ml}$ ácido fosfórico al $17 \%]$. Se agitó por vórtex y se midió a $540 \mathrm{~nm}$. Todas las extracciones se realizaron por triplicado. 


\section{Tratamiento de aguas residuales del lavado de café}

Para los tratamientos se utilizó agua proveniente del tanque del proceso de lavado del café, la cual fue recolectada en envases plásticos de $20 \mathrm{~L}$ y almacenada a -20 ㄷ C hasta el momento de su uso. Para limpiar las aguas residuales con microalgas se realizaron 2 experimentos con los siguientes tratamientos:

Experimento 1 (Parachlorella kessleri)

- Agua residual al $100 \%+P$. kessleri

- Agua residual al $50 \%+P$. kessleri

- Agua residual al $25 \%+P$. kessleri

Experimento 2 (Desmodesmus armatus)

- Agua residual al $100 \%+$ D. armatus

- Agua residual al $50 \%+D$. armatus

- Agua residual al $25 \%+D$. armatus

Las aguas residuales se filtraron con ayuda de una bomba al vacío, usando filtros de 0,45 $\mu \mathrm{m}$. Seguidamente, estas fueron esterilizadas durante 20 minutos a 120 ㅇ C y 15 PSI. Las diluciones del agua residual se realizaron con agua destilada desionizada. La unidad experimenta fue un frasco de vidrio $2,5 \mathrm{~L}$, al que se añadió $1,85 \mathrm{~L}$ de agua residual y $0,15 \mathrm{~L}$ de inóculo de alga, para una concentración final de algas de $1 \times 10^{6}$ células $/ \mathrm{mL}$.

Las condiciones de cultivo fueron irradiancia de 300 $\mu \mathrm{mol}$ de fotones $\mathrm{m}^{-2} \mathrm{~s}^{-1}$, fotoperíodo de 12:12 horas luz/oscuridad, $25 \stackrel{\circ}{\circ} \mathrm{C}+/-3$ 으 y burbujeo permanente con aire del ambiente. Los cultivos se mantuvieron por 15 días.

Para cada tratamientos se determinó DQO, fósforo total y nitrógeno total. Las mediciones se hicieron al inicio y final del cultivo, con el fin de determinar el efecto de las algas en la limpieza de aguas residuales del proceso de producción de café.

\section{Análisis estadístico}

Se realizó una estadística descriptiva, determinando la media para el resultado de cada tratamiento con su respectiva desviación estándar. Para los experimentos de limpieza de agua la unidad experimental fueron recipientes con 1,8 $\mathrm{L}$ de medio, luego de 4 repeticiones por tratamiento.

\section{RESULTADOS Y DISCUSIÓN}

\section{Obtención de cepas unialgal}

Se obtuvieron dos cepas de microalgas de la clase Chlorophyceae:

- Cepa 1: Parachlorella kessleri (Fott \& Nováková) Krienitz, E.H.Hegewald, Hepperle, V.Huss, T.Rohr \& M.Wolf 2004 (figura 1).

Figura 1. Parachlorella kessleri (1.000x)

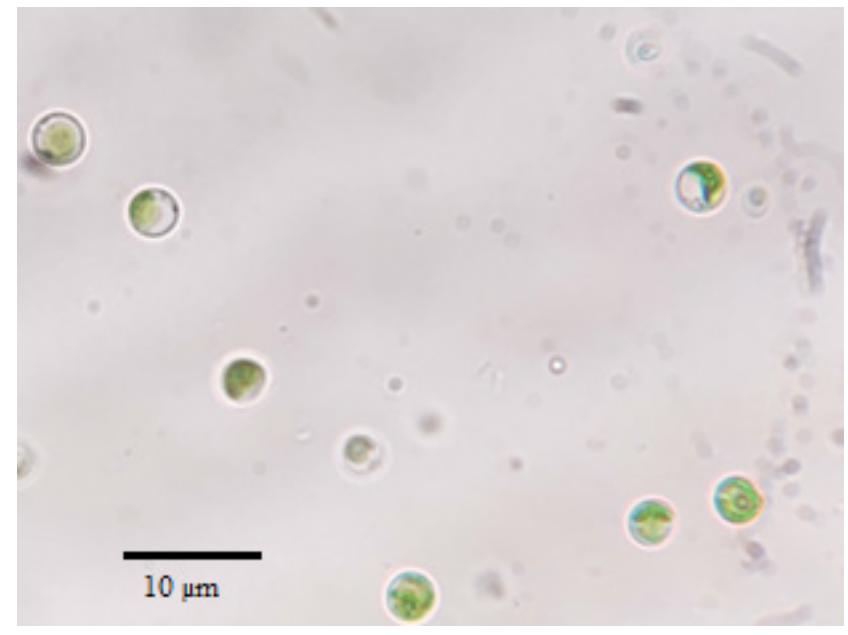

Fuente: Luis Carlos Montenegro Ruíz.

La secuencia que se obtuvo del gen ribosomal 18s fue:

CCAGTAGTCATATGCTTGTCTCAAAGATTAAGCCATGCATGTCTAAGTATAAACTGCTTTATACTGTGAAACTGCGAATGGCTCATTAAATCAGTTATAGTTTATTTGATG GTACCTTACTACCGGATAACCGTAGTAATTCTAGAGCTAATACGTGCGTAAAYCCCGACTYCTGGAAGGGRCGTATTTATTAGATTTAAGGCCGACCCGGCTCTGCCGGTCTCGCGGTGAATCATGATAACTTCACGAATCGCATGGCCTTGCGCCGGCGATGTTTCATTCAAATTTCTGCCCTATCAACTTTCGATGGTAGGATAGAGGCCTACCATGGTGGTAACGGGTGACGGAGGATTAGGGTTCGATTCCGGAGAGGGAGCCTGAGAAACGGCTACCACATCCAAGGAAGGCAGCAGGCGCGCAAATTACCCAATCCTGACACAGGGAGGTAGTGACAATAAATAACAATACCGGGCCTTTTCAGGTCTGGTAATTGGAATGAGTACAATCTAA

El resultado del Blast se muestra en la tabla 1. 
Tabla 1. Resultado del Blast para la cepa 1

\begin{tabular}{|l|c|c|c|}
\hline Accesión & Nombre & Porcentaje de identidad & Fuente \\
\hline AB162911.1 & Parachlorella kessleri & $99,41 \%$ & Hoshina et al. (2004) \\
\hline AB080309.1 & Parachlorella kessleri & $99,41 \%$ & Yamamoto et al. (2003) \\
\hline LC473527.1 & Parachlorella kessleri & $99,41 \%$ & Sasaki et al. (2020) \\
\hline
\end{tabular}

Fuente: elaboración propia.

- Cepa 2: Desmodesmus armatus (Chodat) E.H.Hegewald 2000 (figura 2).

Figura 2. Desmodesmus armatus (1.000x)

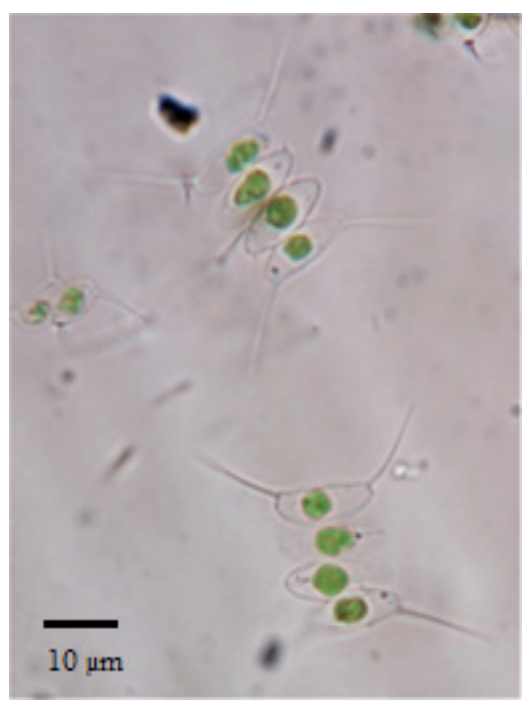

La secuencia que se obtuvo del gen ribosomal 18s fue:

GATCCTGCCAGTAGTCATATGCTTGTCTCAAAGATTAAGCCATGCATGTCTAAGTATAAACTGCTTATACTGTGAAACTG CGAATG G CTCATTAAATCAGTTATAGTTTATTTGGTGGTACCTTCTTACTCGGAATAACCGTAAGAAATTTAGAGCTAATACGTGCGTAAATCCCGACTTCTGGAAGGGACGTATATATTAGATAAAAGGCCGACCGGGCTCTGCCCGACCCGCGGTGAATCATGATATCTTCACGAAGCGCATGGCCTTGTGCCGGCGCTGTTCCATTCAAATTTCTGCCCTATCAACTTTCGATGGTAGGATAGAGGCCTACCATGGTGGTAACGGGTGACGGAGGATTAGGGTTCGATTCCGGAGAGGGAGCCTGAGAAACGGCTACCACATCCAAGGAAGGCAGCAGGCGCGCAAATTACCCAATCCTGATACGGGGAGGTAGTGACAATAAATAACAATACCGGGCATTTCATGTCTGGTAATTGGAATGAGTACAATCTAAATCCCTTAACGAGGATCCATTGGA

El resultado del Blast para esta cepa se muestra en la tabla 2.

Fuente: Luis Carlos Montenegro Ruíz.

Tabla 2. Resultado del Blast para la cepa 2

\begin{tabular}{|l|c|c|c|}
\hline Accesión & Nombre & Porcentaje de identidad & Fuente \\
\hline KP281291.1 & Desmodesmus armatus & $100 \%$ & Westblade et al. (2015) \\
\hline MK541733.1 & Desmodesmus armatus & $100 \%$ & Rad-Menendez (2019) \\
\hline
\end{tabular}

Fuente: elaboración propia. 


\section{Análisis bioquímicos de las cepas de $D$. armatus y $P$. kessleri}

Se encontró que bajo condiciones de cultivo óptimas, en el día 10, durante la fase estacionaria, la composición bioquímica de $D$. armatus fue: proteínas 493,33 $\mathrm{mg} / \mathrm{g}$ peso seco, carbohidratos $310,01 \mathrm{mg} / \mathrm{g}$ peso seco y lípidos 166,66 mg/g peso seco. Para P. kessleri la composición fue: proteínas $396,66 \mathrm{mg} / \mathrm{g}$ peso seco, carbohidratos $320,03 \mathrm{mg} / \mathrm{g}$ peso seco y lípidos 255,05 $\mathrm{mg} / \mathrm{g}$ peso seco (figura 3 ).

Los resultados obtenidos muestran que $D$. armatus y $P$. kessleri tienen contenidos de proteínas, carbohidratos y lípidos similares a los reportados para las especies de los géneros Chlorella, Parachlorella, Desmodesmus y Scenedesmus (Gómez et al., 2017; Palomino et al., 2019; Serrano et al., 2020). Además, se destaca el alto contenido de proteínas en $D$. armatus y el alto contenido de lípidos en $P$. kessleri, dando buenas expectativas para su uso biotecnológico en diferentes sistemas.

Figura 3. Contenido de lípidos, carbohidratos y proteínas en $D$. armatus y $P$. kessleri cultivadas bajo condiciones óptimas
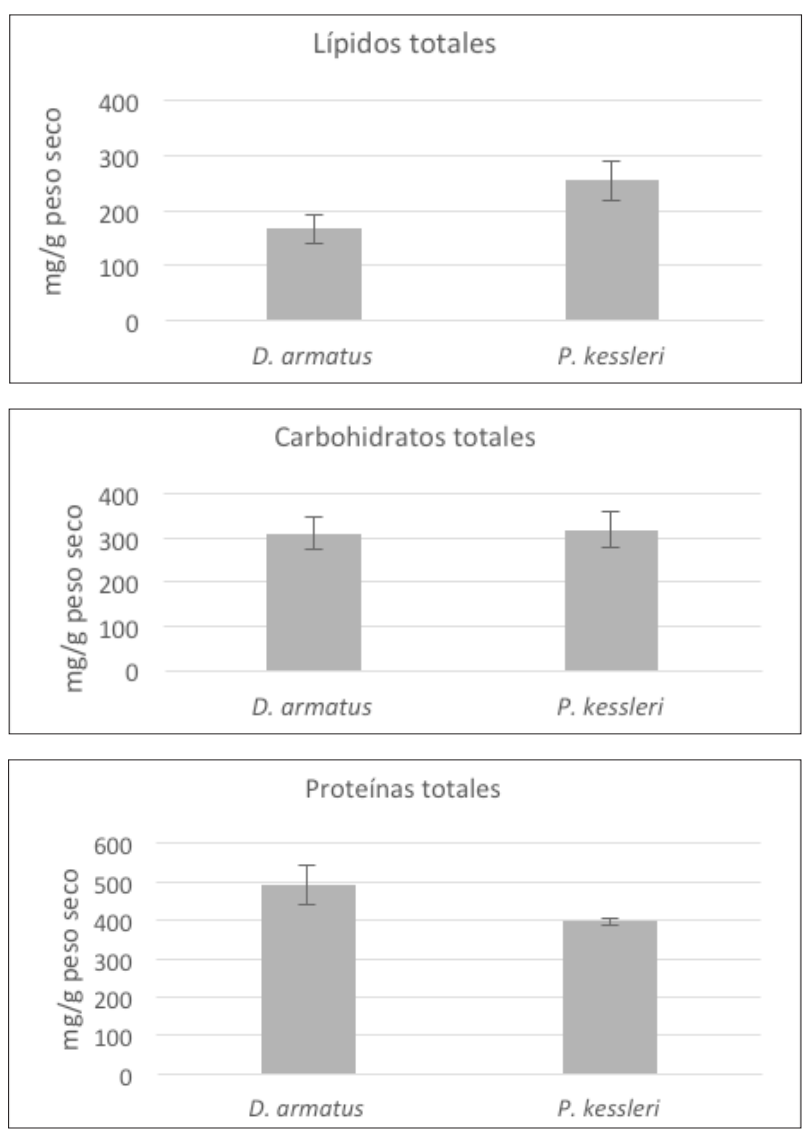

Fuente: elaboración propia.
Las microalgas con alta producción de lípidos son consideradas como materia prima de tercera generación para la producción de biocombustibles (Palomino et al., 2019; Serrano et al., 2020). Los altos contenidos de proteína pueden ser aprovechados para el consumo humano o la elaboración de alimentos concentrados para animales (Chew et al., 2017; González-Delgado et al., 2017; Guccione et al., 2014). Además, se ha encontrado actividad antimicrobiana en varias proteínas en géneros de Parachlorella y Chorella (Yu-Cheng et al., 2019), por lo que otro mercado importante es el de pigmentos obtenidos a partir de estas algas, los cuales son utilizados en la industria alimenticia o nutraceútica como antioxidantes (Kulkarni \& Nikolov 2014; Pozzobona et al., 2020; Safi et al., 2014).

Como se mencionó, después del tratamiento de aguas residuales, la biomasa de microalgas resultante se puede convertir en múltiples productos con alto valor agregado. Las proteínas corresponden a uno de los principales constituyentes de las microalgas y representan entre 50 y $70 \%$, junto con rendimientos en carbohidratos y grasas con valores cercanos a 40 y $30 \%$ de la biomasa en peso seco, respectivamente (Alavijeh et al., 2020); esto, en función de las especies utilizadas y la fase de crecimiento del cultivo al momento de la cosecha. En el género Chlorella sp., cercano a Parachlorella, algunos estudios han realizado aproximaciones a sus rendimientos de macromoléculas, obteniendo porcentajes en aguas residuales de $51-63 \%$ de contenido proteico, $12-17 \%$ de carbohidratos y $14-22 \%$ de lípidos (Mtaki et al., 2021). Por otro lado, en el género Desmodesmus sp. se reportan rendimientos de $11-25 \%$ de proteína, $17-37 \%$ de carbohidratos (Sun et al., 2020) y 26-60 \% de lípidos (Chen et al., 2020; Sun et al., 2020; Zhang et al., 2020). A partir de estos hallazgos, se evidencia que el tratamiento de aguas residuales con microalgas puede tener un doble propósito, puesto que también contribuye a reducir la huella hídrica en algunos procesos productivos (Chen et al., 2020).

\section{Remoción de nutrientes de aguas residuales del café}

Se observó que $P$. kessreli y $D$. armatus son eficientes para la remoción de nitrógeno en aguas residuales, sin importar la concentración inicial de la misma. P. kessreli remueve 70, 67 y $61 \%$ del nitrógeno de las aguas al 100,50 y $25 \%$ de concentración, respectivamente. 
Por su parte, D. armatus remueve 38,70 y $50 \%$ del nitrógeno al 100,50 y $25 \%$ de concentración, respectivamente (figuras 4 y 5 ).

Figura 4. Disminución del contenido de nitrógeno total en aguas residuales del lavado de café utilizando $P$. kessreli durante 15 días de tratamiento

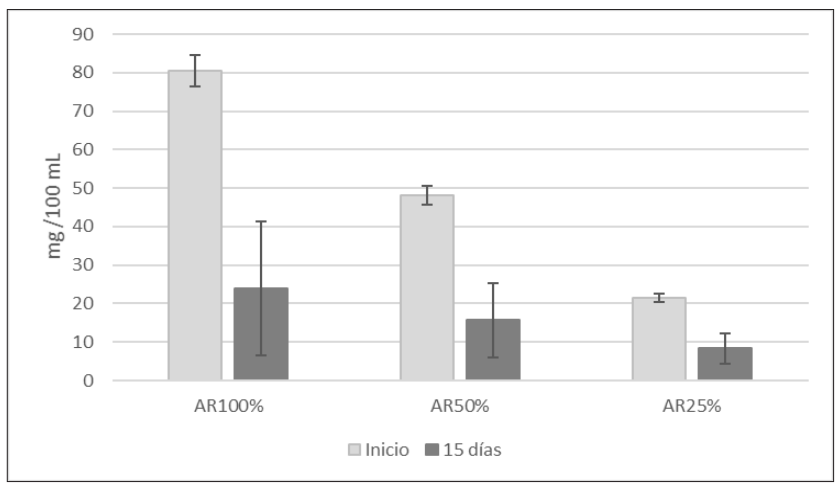

Fuente: elaboración propia.

Figura 5. Disminución del contenido de nitrógeno total en aguas residuales del lavado de café utilizando $D$. armatus durante 15 días de tratamiento

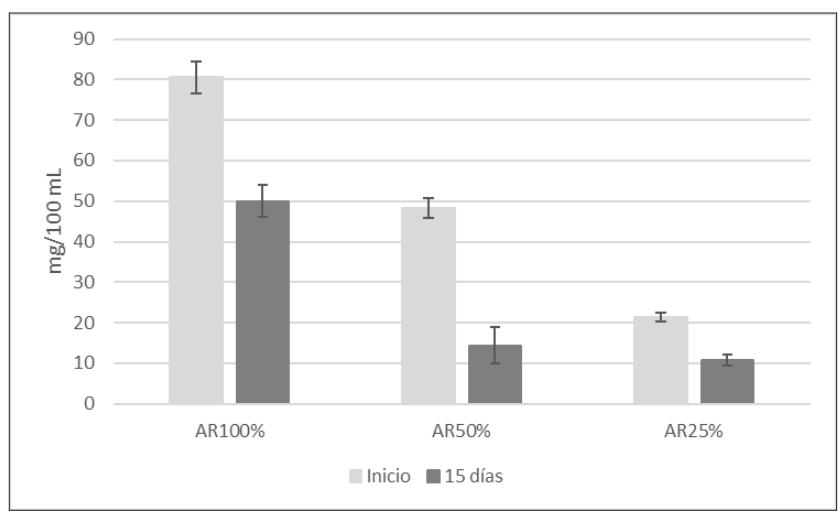

Fuente: elaboración propia.

De otro lado, lo resultados muestran que $D$. armatus es más eficiente en la remoción de fósforo que $P$. kessreli. Para ambas especies, la capacidad de consumir fósforo es baja a bajas concentraciones de agua residual, puesto que $P$. kessreli remueve 15,42 y $15 \%$ del fósforo al 100, 50 y $25 \%$ de concentración, respectivamente, mientras que $D$. armatus remueve 77,87 y $38 \%$ de este elemento al 100,50 y $25 \%$ de concentración, respectivamente (figuras 6 y 7 ).
Figura 6. Disminución del contenido de fósforo total en aguas residuales del lavado de café utilizando $P$. kessreli durante 15 días de tratamiento

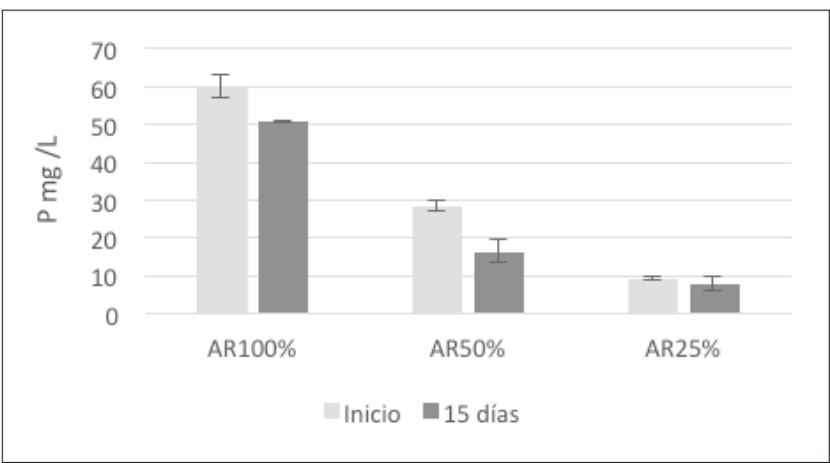

Fuente: elaboración propia.

Figura 7. Disminución del contenido de fósforo total en aguas residuales del lavado de café utilizando $D$. armatus durante 15 días de tratamiento

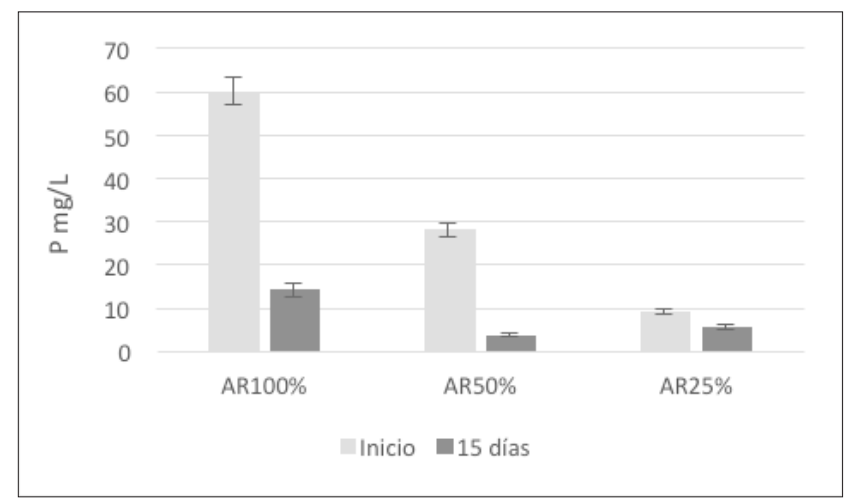

Fuente: elaboración propia.

También se observó que $P$. kessreli es más eficiente disminuyendo la DQO que $D$. armatus, alcanzado cifras de remoción entre 60 y $70 \%$, de acuerdo con la concentración inicial. Por su parte, D. armatus solo disminuyó la DQO un 50 \% a baja concentración, sin alcanzar valores importantes en altas concentraciones de agua residual (figuras 8 y 9 ). 
Figura 8. Disminución de la DQO en aguas residuales del lavado de café utilizando $P$. kessreli durante 15 días de tratamiento

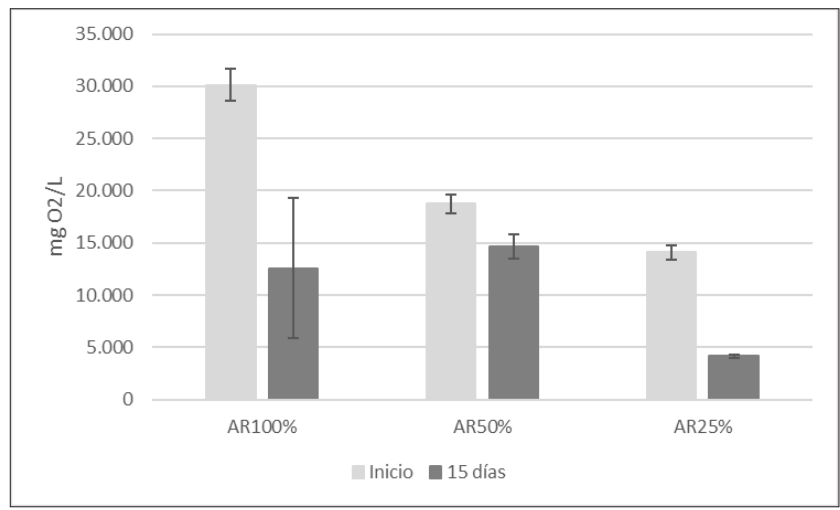

Fuente: elaboración propia.

Figura 9. Disminución de la DQO en aguas residuales del lavado de café utilizando $D$. armatus durante 15 días de tratamiento

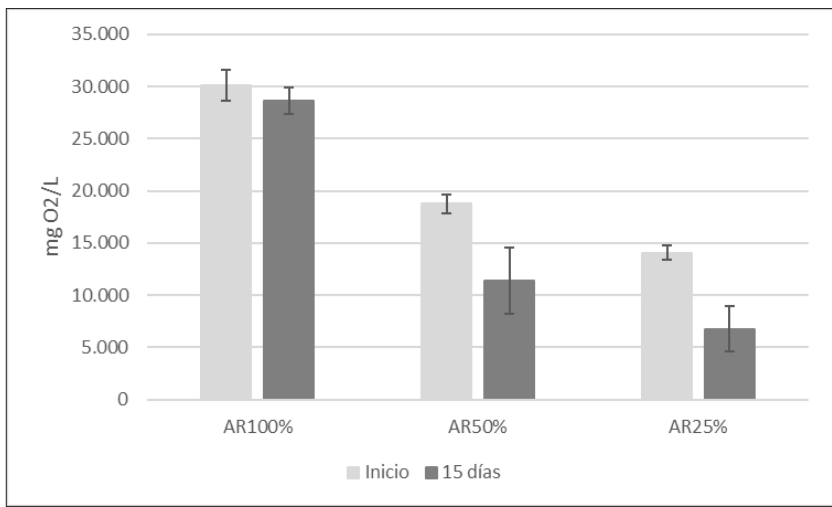

Fuente: elaboración propia.

Las aguas residuales producto de las industrias agropecuarias se caracterizan por altas cargas de materia orgánica, nitrógeno amoniacal y fósforo. Estos compuestos constituyen un factor crítico para problemáticas ambientales como la erosión del suelo, la contaminación atmosférica producto de la volatilización de amoníaco y sulfuro de hidrógeno y la eutrofización de cuerpos de agua (Mousavi et al., 2020; Wang et al., 2020). En la actualidad, las aguas residuales se suelen someter a procesos oxidativos en lagunas para luego ser tratadas en lagunas anaeróbicas y, como etapa final, en lagunas aérobicas facultativas o reactores de película con lecho móvil (Wang et al., 2020), donde las microalgas son las protagonistas, ya que no solo gobiernan el consumo de $\mathrm{CO}_{2}$ sino que también brindan una alternativa segura y rentable a la aireación mecánica (Chen et al., 2020). Las microalgas juegan un papel esencial en estas últimas etapas, demostrando una notable capacidad de absorber nitrógeno y fósforo inorgánicos de forma eficiente, puesto que en este punto los contaminantes han sido degradados y mineralizados por acción de microorganismos heterotróficos (Nur \& Buma, 2019; Wang et al., 2020).

Especies de algas como Scenedesmus obliquus y Chlorella sp. han sido estudiadas para el tratamiento de aguas residuales, generando resultados prometedores en la eliminación de DQO, P y N, con rendimientos aproximados para el género Desmodesmus $s p$. de $92,9 \%$ para amonio y $88,5 \%$ para nitrógeno total, así como remociones de $40,8 \%$ para fósforo y $61,9 \%$ para carbono orgánico disuelto (COD) en 14-17 días de tratamiento (Chen et al., 2020; Ferreira et al., 2017). Por otro lado, Chlorrella sp. reporta rendimientos para remoción de nitrógeno total de $95-100 \%$, fósforo de $85-95 \%$ y COD de $90 \%$ en 14-24 días de tratamiento (Chen et al., 2020; Sepehri et al., 2020), resultados similares a los encontrados en el presente trabajo.

\section{CONCLUSIONES}

Las microalgas Parachlorella kessreli y Desodesmus armatus son eficientes en la depuración de aguas residuales provenientes del lavado del café. Nuestros hallazgos muestran que en 15 días estas algas disminuyen el contenido de sustancias nitrogenadas y fosfatos en cifras superiores a $50 \%$, comprobando su potencial para los sistemas rurales de tratamiento de aguas contaminadas con características similares a los analizados en esta investigación.

Aunque existe una gran diversidad de microalgas con alto potencial para el tratamiento de aguas residuales, son pocas las que permiten su aislamiento y cultivo in vitro. En el presente estudio se alcanzó a tener dos cepas de clorofíceas Parachlorella kessreli y Desodesmus armatus.

Debido al alto contenido de lípidos de Parachlorella kessreli se recomienda su uso para la producción de biocombustibles o alimento para animales, mientras que Desodesmus armatus resulta ser adecuada para la alimentación humana y animal, gracias a su alta producción de proteína. 


\section{AGRADECIMIENTOS}

Los autores agradecen al Servicio de Aprendizaje Nacional (SENA), convocatoria SENNOVA 2020.

\section{REFERENCIAS}

Alavijeh, R. S., Karimi, K., Wijffels, R. H., vanden-Berg, C., \& Eppink, M. (2020). Combined bead milling and enzymatic hydrolysis for efficient fractionation of lipids, proteins, and carbohydrates of Chlorella vulgaris microalgae. Bioresource Technology, 309, 123321. https://doi. org/10.1016/j.biortech.2020.123321

Andersen, R. (2005). Algal culturing techniques. Elsevier Academic Press.

Ardila, L., Godoy, R. D., \& Montenegro, L. C. (2017). Sorption capacity measurement of Chlorella vulgaris and Scenedesmus acutus to remove chromium from tannery wastewater. IOP Conf. Series: Earth and Environmental Science, 83, 012031. https://doi. org/10.1088/1755-1315/83/1/012031

Arias, D. M., Uggetti, E., García-Galán, M. J., \& García J. (2017). Cultivation and selection of cyanobacteria in a closed photobioreactor used for secondary effluent and digestate treatment. Science of The Total Environment, 587-588, 157-167. https://doi.org/10.1016/j. scitotenv.2017.02.097

Ashenafi, F. (2019). Treatment of wastewater effluent from coffee industry using anaerobic mixed culture pseudomonas florescence and Escherichia coli bacteria and with gypsum: A case in DillaEthiopia (Tesis de maestría, Addis Ababa Institute of Technology). Repositorio Addis Ababa Institute of Technology.

Bradford, M. M. (1976). A rapid and sensitive method for the quantitation microgram quantities of protein utilizing the principle of protein-dye binding. Crop Journal, 72, 248-254. https://doi. org/10.1016/j.cj.2017.04.003

Byreddy, A., Gupta, A., Barrow, C., \& Puri, M. (2016). A quick colorimetric method for total lipid quantification in microalgae. Journal of
Microbiological Methods, 125, 28-32. https://doi. org/10.1016/j.mimet.2016.04.002

Cañizares-Villanueva, R. O., Ramos A., Corona, A. I., Monroy, O., de-la-Torre, M., Gomez-Lojero, C., \& Travieso L. (1994). Phormidium treatment of anaerobically treated swine wastewater. Water Research, 9(28), 1891-1895. https://doi. org/10.1016/0043-1354(94)90164-3

Chen, C. Y., Kuo, E. W., Nagarajan, D., Ho, S. H., Dong, C. D., Lee, D. J., \& Chang, J. S. (2020). Cultivating Chlorella sorokiniana AK-1 with swine wastewater for simultaneous wastewater treatment and algal biomass production. Bioresource Technology, 302, 122814. https://doi.org/10.1016/j. biortech.2020.122814

Chen, Z., Shao, S., He, Y., Luo, Q., Zheng, M., Zheng, M., Chen, B., \& Wang, M. (2020). Nutrients removal from piggery wastewater coupled to lipid production by a newly isolated selfflocculating microalga Desmodesmus sp. PW1. Bioresource Technology, 302, 122806. https://doi. org/10.1016/j.biortech.2020.122806

Cheng, Y. S., Zheng, Y., \& Vander-Gheynst, J. S. (2011). Rapid quantitative analysis of lipids using a colorimetric method in a microplate format. Lipids, 46(1), 95-103. https://doi.org/10.1007/ s11745-010-3494-0

Chew, K. W., Yap, J. Y., Show, P. L., Suan, N. H., Juan, J. C., Ling, T. C., Lee, D. J., \& Chang, J. S. (2017). Microalgae biorefinery: High value products perspectives. Bioresource Technology, 229, 53-62. https://doi.org/10.1016/j.biortech.2017.01.006

DuBois, M., Gilles, K., Hamilton, J., Rebers, P., \& Smith, F. (1956). Colorimetric method for determination of sugars and related substances. Analytical Chemistry, 28(3), 350-356. https://doi. org/10.1021/ac60111a017

Ferreira, A., Ribeiro, B., Marques, P. A. S., Ferreira, A. F., Dias, A. P., Pinheiro, H. M., Reis, A., \& Gouveia, L. (2017). Scenedesmus obliquus mediated brewery wastewater remediation and $\mathrm{CO}_{2}$ biofixation for green energy purposes. Journal of Cleaner Production, 165, 1316-1327. https://doi. org/10.1016/j.jclepro.2017.07.232 
Gerchakov, S., \& Hatcher, P. (1972). Improved technique for analysis of carbohydrates in sediments. Limnology and Oceanography, 17(6), 938-943. https://doi.org/10.4319/ lo.1972.17.6.0938

Gómez-Pérez, C., Espinosa, J. J., Montenegro, L. C., \& Van-Boxtela, J. B. (2017). Twisted tubular photobioreactor fluid dynamics evaluation for energy consumption minimization. Algal Research, 27, 65-72. https://doi.org/10.1016/j. algal.2017.08.019

González-Delgado, A. D., Barajas-Solano, A., \& ArdilaÁlvarez, A. M. (2017). Producción de biomasa y proteínas de Chlorella vulgaris Beyerinck (Chlorellales:Chlorellaceae) a través del diseño de medios de cultivo selectivos. Corpoica Ciencia y Tecnología Agropecuaria, 18(3). https://doi. org/10.21930/rcta.vol18_num3_art:736

Gorain, P. C., Paul, I., Singh, P., \& Pala, R. (2019). An integrated approach towards agricultural wastewater remediation with fatty acid production by two cyanobacteria in bubble column photobioreactors. Algal Research, 42, 101-594. https://doi.org/10.1016/j. algal.2019.101594

Guccione, A., Biondi, N., Sampietro, G., Rodolfi, L., Bassi, N., \& Tredici, M. R. (2014). Chlorella for protein and biofuels: from strain selection to outdoor cultivation in a green wall panel photobioreactor. Biotechnology for Biofuels, 7, 84. https://doi.org/10.1186/1754-6834-7-84

Hoshina, R., Kamako, S. I., \& Imamura, N. (2004). Phylogenetic position of endosymbiotic green algae in Paramecium bursaria Ehrenberg from Japan. Plant Biology, 6(4), 447-453. https://doi. org/10.1055/s-2004-820888

Jenniffer, A. A., Chandran, T., Muthunarayanan, V., Ravindran, B., Nguyen, V. K., Nguyen, X. C., Bui, X. T., Ngo, H. H., Nguyen, X. H., Chang, S. W., \& Nguyen, D. D. (2020). Evaluation of efficacy of indigenous acidophile- bacterial consortia for removal of pollutants from coffee cherry pulping wastewater. Bioresource Technology Reports, 11(September), 100533. https://doi. org/10.1016/j.biteb.2020.100533
Kulkarni, S., \& Nikolov, Z. (2018). Process for selective extraction of pigments and functional proteins from Chlorella vulgaris. Algal Research, 35, 185193. https://doi.org/10.1016/j.algal.2018.08.024

Lynch, F., Santana-Sánchez, A., Jämsä, M., Sivonen, K., Aro, E. A., \& Allahverdiyeva, Y. (2015). Screening native isolates of cyanobacteria and a green alga for integrated wastewater treatment, biomass accumulation and neutral lipid production. Algal Research, 11, 411-420. https:// doi.org/10.1016/j.algal.2015.05.015

Melgarejo, L. (2010). Experimentos en fisiología vegetal. Universidad Nacional de Colombia.

Mishra, S., Suh, W., Farooq, W., Moon, M., Shrivastav, A., Park, M., \& Yang, J. (2014). Rapid quantification of microalgal lipids in aqueous medium by a simple colorimetric method. Bioresource Technology, 155, 330-333. https:// doi.org/10.1016/j.biortech.2013.12.077

Mousavi, S. A., Sarshad, M., \& Soltani, S. (2020). Negative impacts of nomadic livestock grazing on common rangelands' function in soil and water conservation. Ecological Indicators, 110, 105946. https://doi.org/10.1016/j.ecolind.2019.105946

Mtaki, K., Kyewalyanga, M. S., \& Mtolera, M. S. P. (2021). Supplementing wastewater with NPK fertilizer as a cheap source of nutrients in cultivating live food (Chlorella vulgaris). Annals of Microbiology, 71(7). https://doi.org/10.1186/ s13213-020-01618-0

Navitha, K. R., \& Kousar, H. (2018). A comparative study on the potential of Aspergillus niger and Aspergillus flavus for the treatment of coffee processing effluent. International Journal of Environment, Ecology, Family and Urban Studies, 8(4), 17-22. https://doi.org/10.24247/ ijeefusaug20182

Nur, M. M. A., \& Buma, A. G. J. (2019). Opportunities and challenges of microalgal cultivation on wastewater, with special focus on palm oil mill effluent and the production of high value compounds. Waste and Biomass Valorization, 10(8), 2079-2097. https://doi.org/10.1007/ s12649-018-0256-3 
Palomino, A., Montenegro, L. C., \& Godoy, R. D. (2019). Evaluation of yield-predictive models of biocrude from hydrothermal liquefaction of microalgae. Algal Research, 44, 101669. https:// doi.org/10.1016/j.algal.2019.101669

Pires, J. F., Viana, D. C., Braga, R. A., Schwan, R. F., \& Silva, C. F. (2021). Protocol to select efficient microorganisms to treat coffee wastewater. Journal of Environmental Management, 278(P2), 111541. https://doi.org/10.1016/j. jenvman.2020.111541

Pozzobona, V., Levasseura, W., Guerina, C., GaveauVaillantb, N., Pointchevala, M., \& Perréa, P. (2020). Desmodesmus sp. pigment and FAME profiles under different illuminations and nitrogen status. Bioresource Technology Reports, 10, 100409. https://doi.org/10.1016/j. biteb.2020.100409

Rodriguez, N. V., Sanz, J. R., Oliveros, C. E., \& Ramirez, C. G. (2015). Beneficio del café en Colombia. Federación Nacional de Cafeteros/Cenicafé.

Safi, C., Camy, S., Frances, C., Montero-Varela, M., Calvo-Badia, E., Pontalier, P., \& Vaca-Garcia C. (2014). Extraction of lipids and pigments of Chlorella vulgaris by supercritical carbon dioxide: influence of bead milling on extraction performance. Journal of Applied Phycology, 26, 1711-1718. https://doi.org/10.1007/ s10811-013-0212-3

Sepehri, A., Sarrafzadeh, M. H., \& Avateffazeli, M. (2020). Interaction between Chlorella vulgaris and nitrifying-enriched activated sludge in the treatment of wastewater with low $\mathrm{C} / \mathrm{N}$ ratio. Journal of Cleaner Production, 247, 119164. https://doi.org/10.1016/j.jclepro.2019.119164

Serrano, L. M., Montenegro, L. C., \& Godoy, R. D. (2020). Effect of $\mathrm{CO}_{2}$ aeration, irradiance, and photoperiod on biomass and lipid accumulation in a microalga autotrophically cultured and selected from four Colombian-native strains. Bioresource Technology Reports, 12, 100578. https://doi.org/10.1016/j.biteb.2020.100578
Sun, X., Li, P., Liu, X., Wang, X., Liu, Y., Turaib, A., \& Cheng, Z. (2020). Strategies for enhanced lipid production of Desmodesmus sp. mutated by atmospheric and room temperature plasma with a new efficient screening method. Journal of Cleaner Production, 250, 119509. https://doi. org/10.1016/j.jclepro.2019.119509

Wang, Y., Wang, S., Sun, L., Sun, Z., \& Li, D. (2020). Screening of a Chlorella-bacteria consortium and research on piggery wastewater purification. Algal Research, 47, 101840. https://doi. org/10.1016/j.algal.2020.101840

Westblade, L. F., Ranganath, S., Dunne, W. M., Burnham, C. A., Fader, R., \& Ford, B. A. (2015). Infection with a chlorophyllic eukaryote after a traumatic freshwater injury. The New England Journal of Medicine, 372(10), 982-984.

Wu, L., Zhu, Q., Yang, L., Li, B., Hu, C., \& Lan S. (2018). Nutrient transferring from wastewater to desert through artificial cultivation of desert cyanobacteria. Bioresource Technology, 247, 947-953. https://doi.org/10.1016/j. biortech.2017.09.127

Yamamoto, M., Nozaki, H., Miyazawa, Y., Koide, T., \& Kawano, S. (2003). Relationship between presence of a mother cell wall and speciation in the unicellular microalga Nannochloris (Chlorophyta). Phycology, 39, 172-184. https:// doi.org/10.1046/j.1529-8817.2003.02052.x

Zhang, Y., Huang, Z., Zheng, H., Wang, Q., \& Li, A. (2020). Growth, biochemical composition and photosynthetic performance of Scenedesmus acuminatus under different initial sulfur supplies. Algal Research, 45, 101728. https://doi. org/10.1016/j.algal.2019.101728 